\title{
On the Implementation and Evaluation of Berkeley Sockets on Maestro2 cluster computing environment
}

\author{
Ricardo Guapo ${ }^{12}$, Shinichi Yamagiwa ${ }^{1}$ and Leonel Sousa ${ }^{12}$ \\ ${ }^{1}$ INESC-ID, Rua Alves Redol 9 Apartado 13069, 1000-029 Lisboa Portugal \\ ${ }^{2}$ Instituto Superior Técnico - Av. Rovisco Pais, 1049-001 Lisboa Portugal \\ \{guapo, yama, las\}@ sips.inesc-id.pt
}

\begin{abstract}
The support on cluster environments of "legacy protocols" is important to avoid rewriting the code of applications, but this support should not prevent to achieve the maximum communication performance. This paper addresses this issue by implementing the Berkeley sockets interface over the MMP message passing protocol, which is the lower layer of the Maestro2 cluster communication network. Experimental results show that MMP-Sockets offers a minimum latency of $25 \mu \mathrm{s}$ and a maximum throughput of $1250 \mathrm{Mbps}$. These values correspond to a relative increase of $80 \%$ for the latency and a decrease of about $30 \%$ for the throughput, regarding to a communication based only on MMP. However, MMP-Sockets increases the compatibility and portability of developed applications.
\end{abstract}

\section{Introduction}

Performance extracted from off-the-shelf components is the key to design efficient commodity cluster computers. For the parallel application in clusters, it is important to reduce the overheads in the network hardware such as the copy operations between user application and drivers buffers and also the excessive TCP information headers for local communication.

To achieve the potential network performance of cluster computers hardware, dedicated software is applied for implementing the communication interface among application's parallel processes. An example of such specific networks is the Myrinet [10] network hardware and its dedicated low-level protocols GM [1], PM [4] and BIP [8]. GM, PM and BIP optimize the communication with Myrinet network hardware and implement the zero-copy network interface, by touching the user memory space that is locked by the OS (pindowned) directly to send and receive messages. Those protocols touch the hardware directly from the user application to bypass the thick conventional network pro- tocol layers. Thus, those communication software are able to achieve almost $100 \%$ of the potential network hardware performance.

To take advantage of such high performance protocols, the applications need to use those proprietary communication software. Once the necessity to change the platform of the applications, namely to a different cluster, a serious problem arises to keep the compatibility of the code, namely in what respect communication. Therefore, it is necessary for the programmer to rearrange the communication part of the applications regarding to the new dedicated communication software.

To address this portability/compatibility problem of applications on different cluster hardware, the MPI standard was proposed [9]. MPI defines a standard API for a message passing library. It is suitable for parallel applications for which the communication size and timing are defined statically, such as: matrix computation, Fast Fourier Transform (FFT) and a LU decomposition. However, not all high performance communication demanding programs have precise knowledge about the amount data transfers, e.g.: HTTP based video transcoding [6] and large data-bases.

Even if ported to MPI, those kind of applications still require the use of a TCP connection to clients outside the cluster. This forces the existence of two communication methods/libraries, TCP and MPI, to be conjugated together, which can be avoided if TCP would already be present at high performance for inside cluster communication as the standard unified protocol. Therefore, it has been recently started an effort to bring cluster high performance communication to applications using TCP, by implementing libraries which will enable the execution of those applications in clusters [3].

This paper is focused on the design and implementation of a fully compatible communication library of TCP over a dedicated communication software that can achieve high performance communication for cluster computing. For that, the Berkeley sockets interface was implemented over the MMP message passing protocol, which is the lower 
layer of the Maestro2 cluster communication environment. Moreover, we will discuss the variation in the communication performance with the change of the parameters in our socket implementation.

The paper is organized as follows: the next section presents an overview of the high performance network technology Maestro2, with the dedicated communication software called MMP. Section 3 presents the design and implementation of the Berkeley sockets API over the MMP communication library. Section 4 presents experimental results and evaluate the relative performance of the new developed library. Section 4 finally concludes this paper.

\section{Background}

The Maestro2 network [11][12] is an intelligent network that has been developed to address the overheads of intercluster communication. The Maestro network is supported by a dedicated communication protocol, implemented as a message passing library, called MMP.

Maestro2 network: The Maestro2 network is composed by network interfaces (NI) and a Switch Box (SB) as shown in the diagram of figure 1. Each network interface is connected via two Low Voltage Differential Signalling (LVDS) [5] cables for transmission and reception, and is connected to a commodity computer such as a personal computer or a WorkStation via a 64bit@66MHz PCI bus. The connection between NI and SB is full-duplex and the peak bandwidth is of $3.2 \mathrm{Gbps}$. Currently, the SB has ports to directly connect up to eight $\mathrm{NI}(\mathrm{s})$. One or more ports can increase the fan-out of the switch by cascading $\mathrm{SB}(\mathrm{s})$. The NI includes a NI manager, a PCI interface, network FIFO buffers, a link controller (MLC-X) and LVDS transmitter/receiver. The NI manager works as a processor element in charge of handling communications, and consists of a PowerPC603e@300MHz and 64Mbyte of SDRAM. The PCI interface maps the address space of the SDRAM and part of host processor's memory into the PowerPC's address space. A 8Kbyte network FIFO buffers to store incoming and outgoing messages. The MLC-X is a full duplex link layer controller on which the continuous network burst transfer is implemented. MLC-X supports two communication channels between the network FIFO buffers. And finally, LVDS transmitter/receiver drive its physical medium under control of MLC-X. It transmits and receives data via its 3.2 Gbps full duplex link. The PCI interface, network buffers, and MLC-X are implemented into the Virtex-II FPGA (Field Programmable Gate Array) chip [13]. The SB consists of four SB interfaces, a SB manager and a switch controller. Each SB interface manages two ports and includes a message analyzer. The message analyzer extracts the message headers of each incoming message, and passes it to the SB manager. The SB manager consists of a PowerPC603e, 32Mbyte SDRAM, and a routing circuit that

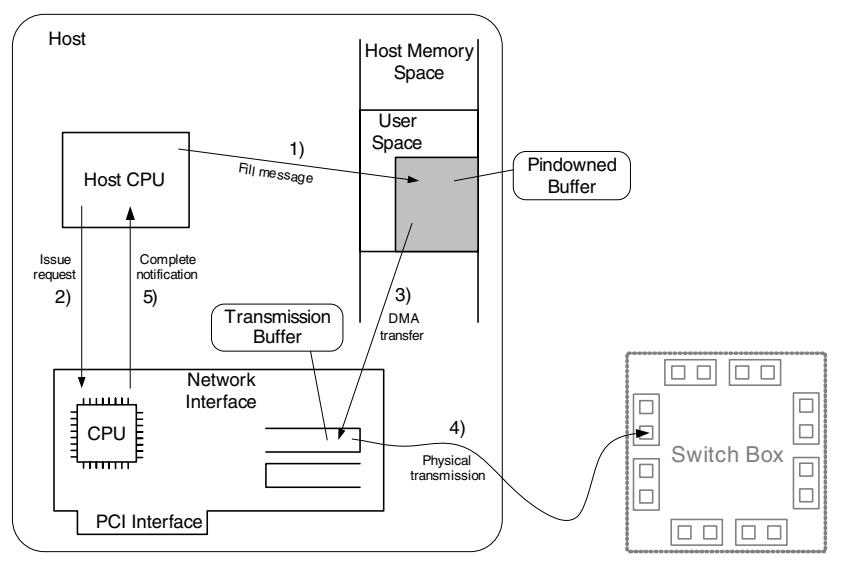

\section{Figure 1. Maestro network diagram and send operation subactions}

generates and writes requests to the switch controller.

Maestro2 Message Passing protocol: To achieve high performance with Maestro2 PC clusters, the low latency and high bandwidth dedicated communication MMP library was developed. MMP consists of a user level library and the Maestro2 communication firmware that directly controls NI and SB. The two main key observations in developing MMP were: $i)$ to avoid unnecessary overhead so that the communication does not degrade the overall performance of the parallel applications; $i i$ in accessing the CPU, computation must have higher priority than communication.

Regarding the first issue, MMP implements the zerocopy mechanism on Linux OS (Operating System), eliminating data copy between user and kernel memory spaces and avoiding system calls. The zero-copy communication technique consists on exchanging messages between the sender's application memory space to the receiver's one directly, by using DMA operations between network hardware buffers and application buffers that are allocated and locked [7] (generally called, pindowned).

To deal with the second issue, MMP communication functions were made to be non-blocking. In addition, complex communication operations, such as the creation of data chunks, migrates to the physical network. Therefore, the communication code will be overlapped with the computation code on the host processor. MMP provides primitive message-based non-blocking communication interface functions (MMP_send (), MMP_Recv ( ) ), and corresponding request completion synchronization functions MMP_send_wait (), MMP_recv_wait () .

MMP uses a connection-less data transmission system based on messages, similar to UDP. Arguments for the sending or receiving functions indicate only the receiver and sender end-point identification, besides message identification parameters. When an application call MMP_Initialize () function, MMP will provide an in- 
dividual "port" to identify the application end-point in the MMP network. In figure 1 it is presented the sub-actions performed by a MMP send operation: 1) application creates the message in the pindowned memory; 2) it notifies the send request; 3) MMP firmware in NI issues DMA transfers between main memory and network buffers; 4) the message is transmitted by link-layer to SB and 5) the request status is updated to completed.

It was on top of MMP library that the Berkeley socket interface was implemented in this work. Next section will be focused on the implementation aspects.

\section{Berkeley Socket Implementation}

The Berkeley Socket compatible communication library was implemented over MMP, (MMP-Sockets), using $\mathrm{C}$ and $\mathrm{C}++$ programming languages.

To consider the full compatibility for the Berkeley Socket, the MMP-Socket must support functions which interact with file descriptors. Due to the lack of the concept of file descriptors in MMP, it is required the design of functions to create and to maintain the file descriptor, in order to keep the compatibility. Dedicated data structures are used to manage all connections and also the data transmissions using MMP while assuring data integrity.

The MMP-Socket will work in the user mode. This allows to improve the performance, because context switching between the user mode and the kernel mode is almost no longer required for communication operations.

\subsection{Structure}

Implementation presents a layered structure, to maximize compatibility, portability and to allow easier future developments, such as porting for other message passing protocols.

MMP-Sockets was implemented using a three stacking layers model, as depicted in gray in figure 2: the upper layer interfaces with the application, providing the API calls; the middle layer, the core engine, accommodates all the required data structures and associated methods to perform the communication tasks; the lowest layer interfaces with MMP and automatizes some MMP procedures.

Inside core engine, methods are provided to access the data structures, which are categorized in two main classes: data managing methods and data consulting methods. Managing methods are intended for insertion, removal or change of information in the data structure, while consulting methods are used to browse, search and retrieve information from the data structure. Managing methods are intended to perform the maintenance of the data required to communicate while consulting methods help in the communication procedure, and for that can be included in the class of transmission methods. The transmission methods class is orthogonal to the managing and consulting classes, including all

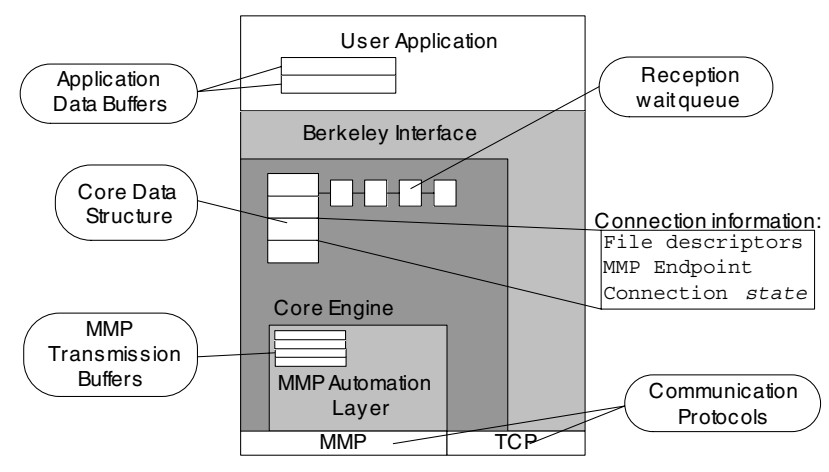

Figure 2. MMP-Sockets layered model and main components

the methods of the consulting class and only the "change of information" maintenance methods from the managing class. Transmission methods can be located at the interface level between core engine and the used transmission protocols, TCP and MMP. Each implementation code is in charge of managing its own data structure, to allow the establishment and the use of the MMP channel, associated with the state. State reveals the capability to use (or not) the MMP channel to transmit the application data. When special events that imply a change in the MMP channel state occur, control messages are exchanged between both communication partners to inform or request a state change.

The interface layer uses the available methods and data structures provided by the core engine layer to fulfill the communication task. When MMP use is disabled, it can interface directly with the TCP protocol. It not only includes the Berkeley sockets API but also fork (), dup (), dup2(), select (), execve(), read() and write ().

The communication operation is assured by the two main transport layers. To assist in MMP communication, there is a middleware layer to automatize some of the MMP more repetitive and demanding tasks (e.g. channel creation). MMP transmission data structures such as request handlers and pindowned buffers are maintained by this layer.

\subsection{Layers}

The core engine presents a main data structure to manage the open connections and the MMP transmissions. The main data structure is a list of records where each record represents an open regular connection with an optional companion MMP channel. The list is implemented using a fixed size array of records elements to avoid memory allocation overheads. In the record, all information relevant to the connection and its status is preserved: the associated file descriptors to the socket, MMP endpoint information and connection state. Each socket connection is uniquely identified by the first associated file descriptor. As send () 
operation is blocking, there is no need for a waiting queue to send data. However, reception is an asynchronous operation and more data can arrive than the requested, so a waiting queue had to be implemented. Data received by the implementation waits in the reception queue until there is an appropriate receive request from the application.

Transmission and reception buffers are required for MMP data transmission. These buffers are managed at the middleware/automation layer. Their size and number was experimentally determined by extensive testing, whose results are presented in the experimental results section. Along with the buffers are the necessary controlling structures, which includes request handlers to manage the MMP transmissions. These structures allow each transmission to be monitored independently and, by that, to use each buffer more efficiently. The MMP automatization layer is responsible to deliver the received MMP messages in order to the upper core engine.

1) Managing methods: Each MMP-Sockets API function, when called, will use the available methods to perform specific tasks, such as: i) resource allocation to initialize the MMP-sockets environment; ii) record insertion, iii) record modification and iv) record removal to manage the open connections list.

i) Resource allocation: The MMP-Sockets environment initialization, e.g. data structures allocation and MMP initialization, is performed when the socket () function is called. Data structures de-allocation and MMP endpoint release are done when application normally exit () or performs the load of another binary image through execve (). Application maintains always the same MMP port, and it only performs MMP initialization and MMP shutdown operations once.

ii) Record insertion: The insertion method in the list of open connections is only performed by accept () and connect () functions. At the initial connecting sequence, the necessary information is exchanged and used to fill an entry in the list. If both end points can use MMP between them, it is registered in the corresponding record. Upon a sending or receiving operation, the record is checked to verify the possibility to use the MMP transmission channel. If MMP is disabled in one of the applications, or the information exchange fails, the establishment of the MMP companion channel is aborted and regular socket will be used for this particular connection.

iii) Record modifiers: There are special interface functions that, when called, represent a special event and might change the state of the MMP channel, such as: fork (), dup ()/dup2 (), close (), shutdown (), execve (). As a result of a fork () operation, a socket gets shared by more than one process, which invalidates the use of MMP for data transmissions, and the normal TCP socket connection is used to exchange data. When one of the parent or child process closes its end, MMP channel use is enabled to the remaining connection. dup () and dup2 () functions duplicate file descriptors, and so it is necessary to keep track of the file descriptors referencing the connections managed by MMP-Sockets. shutdown () effects are registered in the corresponding record to avoid unnecessary transmissions. execve ( ) function forces the complete shutdown of the MMP-Sockets environment: MMP environment resources de-allocation is performed to free the resources while normal TCP sockets are kept open as they will be passed on to the new binary image.

iv) Record removal: The close () function is the only one that clears records in the list of open connections. When an application closes the only file descriptor referring to a socket which has a record in the list, procedures are taken to close the MMP channel along with the socket connection, and the record is removed from the list.

2) Transmission methods: Methods to manage the other data structures, such as waiting queues and transmission buffers, are called when the interface transmission functions send () and recv() are executed. Upon a send () request from an application, the relevant connection record is consulted to confirm the possibility to use the MMP transmission. If MMP transmission is enabled, application data to be sent is packed into the send buffers along with the MMP-Sockets header and transmitted to the receiver. When the amount of data is greater than the payload message size, data is fragmented in several messages. To respond to a recv () request, data is retrieved from the associated waiting queue; if the queue is empty, it is necessary to wait for data to be received.

\subsection{Performance improvements}

The size property of an MMP message is rounded up to a multiple of 32, as the granularity of the Maestro2 transmission system is of 32 Bytes. However, applications send () and recv () calls do not comply with this granularity. In order to not affect the useful payload size, which can decrease performance, the TAG identifying property of MMP messages header is filled with the correct size, so that receiver can ignore stuffing bytes. A performance enhancing strategy was adopted at the MMP reception procedure: to avoid the use of a fixed size for the exchanged messages or a rendez-vous approach to inform the message size, which degrades performance, messages with size equal or less than the expressed in the request are only considered as a match if all other parameters match. This allows to receive any MMP message with size less or equal to the buffer size in any buffer. The use of wildcards for sender endpoint identification parameter allows the use of any buffer in any of the created MMP channels, maximizing the resources' usage. The disadvantages are that the maximum size of the MMP messages is equal to the size of the individual buffers used 


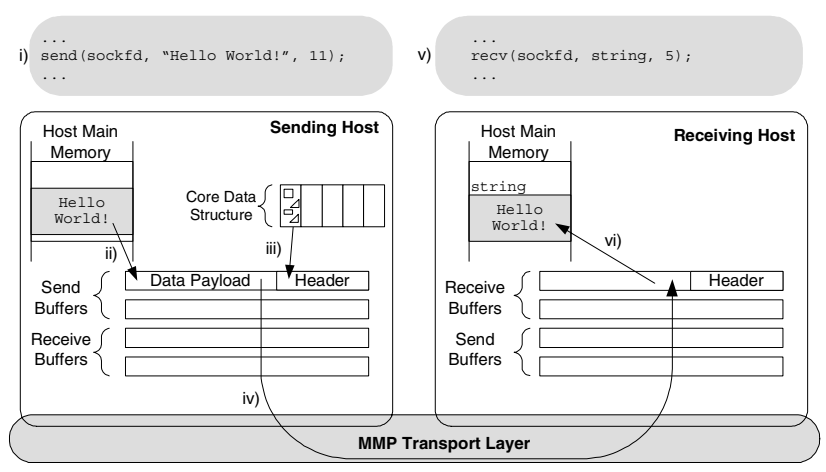

Figure 3. MMP-Sockets Transmission subactions example

and all buffers must be of same size.

\subsection{Transmission procedure}

The following paragraphs detail the steps involved in a complete transmission of data between two applications using the MMP-Sockets communication library.The main actions are depicted in figure 3. It is assumed for the following presentation that both applications successfully completed the MMP-Sockets initialization and were able to establish a connection between them.

When the sender application/process calls the send () function (figure 3, $i$ ), a procedure of filtering the send request is initiated, to check wether the send () can use the MMP protocol. If use of MMP channel is allowed, the transmission is processed by the MMP channel, starting data packing.

Application data is copied and packed in send buffer (ii), along with MMP-Sockets header (iii) and sent to receiver. Each message is sent (iv) issuing an MMP_Send () request and the buffer can be reused when request status is confirmed as completed.

When the receiver application calls recv() function $(v)$, the filtering procedure is also performed. The waiting queue is checked to see if there is already pending data to be delivered: if exists, it is passed to the application being removed from the queue; if not, a wait is performed for a message with data relative to this request. As soon as it is received, the data is delivered to the application (vi).

\section{Results - Performance Evaluation}

This chapter describes the most relevant aspects concerning the experiments performed in order to tune and evaluate the performance of the proposed MMP-Sockets implementation on the Maestro2 system.

The first set of experiments are performed to determine the best values for the buffers parameters, namely quantity and size. These are important parameters: if the buffers are too much large, they consume unnecessary resources and can affect performance, but very small buffers increase the fragmentation and increase transmission overhead, decreasing overall performance. The buffer quantity can also affect performance: too few buffers and the waiting time for resources will surely increase, while a very large quantity of buffers might be a waste of an important resource: physical host memory. The second experiment concerns about the performance loss due to the mandatory copy operations between pindowned buffers (PB) from the new library and the user application buffers (UB). Due to this memory to memory copy operation MMP-Sockets can be classified as a one-copy communication library, and the experiment will evaluate this overhead and conclude how to minimize it. A last experiment present the comparison between the MMP and the MMP-Sockets performance, using the optimum values for the parameters found in the previous experiments. It allows to evaluate the performance loss due to the stacking of the compatibility-increasing library (MMP-Sockets) on top of the high performance communication library (MMP).

\subsection{Tools and Experimental Setup}

The NetPIPE [2] benchmark was used to evaluate the performance of both the MMP communication library and the implemented MMP_Sockets communication library, as well as the memcpy () operation performance inside the Maestro2 hosts. NetPIPE is a communication performance evaluation tool that measures the time taken to migrate a message with a fixed amount of data, while implementing a ping-pong pattern. It also allows to test the system by adding a small perturbation to each fixed size message, to detect anomalies due to buffers boundaries. NetPIPE has a clear-layered architecture offering a common API, which makes its use independently of the communication protocols, e.g. MMP.

The experimental results described in this section were obtained using two Maestro2 hosts with the NICs connected directly to each other. The host machine for the Maestro2 network interfaces consisted in a DUAL 1.0GHz Intel Pentium-3, a Serverworks HE-SL chipset motherboard with a PCI bus of 64 bits working at $66 \mathrm{MHz}$, and 512 Mbytes of SDRAM PC133 as main memory.

\subsection{Buffer Analysis}

During implementation, communication buffers were a key factor for the achievable performance. Experimental analysis allows to discover the best set of values for the number of buffers and their size. The analysis was performed by exhaustively testing several pairs of values, to understand wether they affected the performance independently or not. The couple of values are obtained by continuously varying the number of buffers between 1 and 16 , 


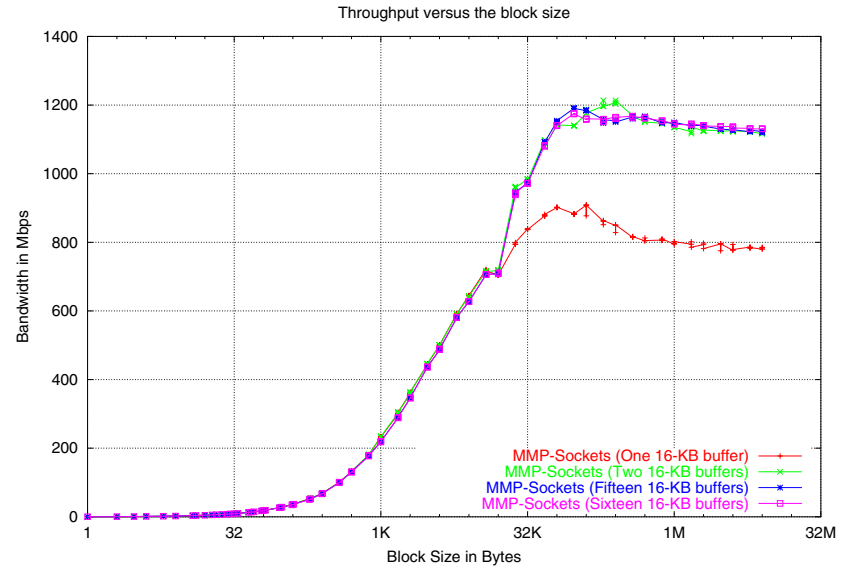

Figure 4. Throughput performance of MMPSockets with 16-KB buffers for 1, 2, 15 and 16 buffers

and the size of the buffers, ranging all the powers of two between $2^{10}$ and $2^{20}$. These numbers represent an individual operation, sending or receiving, as they are similar. Figure 4 presents the most relevant results for multiple runs with the buffer size fixed at $16-\mathrm{KB}$. Lines concerning the buffer quantities from three to fourteen were not drawn because they presented a similar behavior as the lines representing two, 15 and 16 buffers. The observed minimum latency is approximately equal for all buffer quantities, meaning that the buffer quantity does not influence the minimum latency, which is about $25 \mu \mathrm{s}$.

All lines present a similar behavior for data block sizes which do not trigger fragmentation; for data block sizes which does get fragmented, performance differs depending if there is one or multiple PBs. When using more than one buffer, performance increases rapidly when the transmission start to use more than one buffer, due to the pipelining of the transmission stages. To conclude the analysis of figure 4 , it can be noticed that when using only one buffer the performance decays for sizes slightly larger than the sizes for which the peak performance is achieved. This effect is due to the serialization of the time consuming transmission operations (figure 3, ii, iv, vi), as it will be observed in the second experiment. Furthermore, the performance is improved by increasing the number of buffers but with 16 buffers the queue processing overhead already decreases the peak performance. Thus, the optimal buffer quantity for this environment is 15 buffers.

The graphic in figure 5 plots all the lines combining all the buffer sizes (powers of 2) with 4 buffers. All lines present the similar behavior for small messages, and so minimum latency is approximately equal for all buffer quantities, meaning that the buffer size does not influence the minimum latency (about $25 \mu \mathrm{s}$ ). For small PBs sizes, saturation is attained very soon due to the need of fragmentation, and

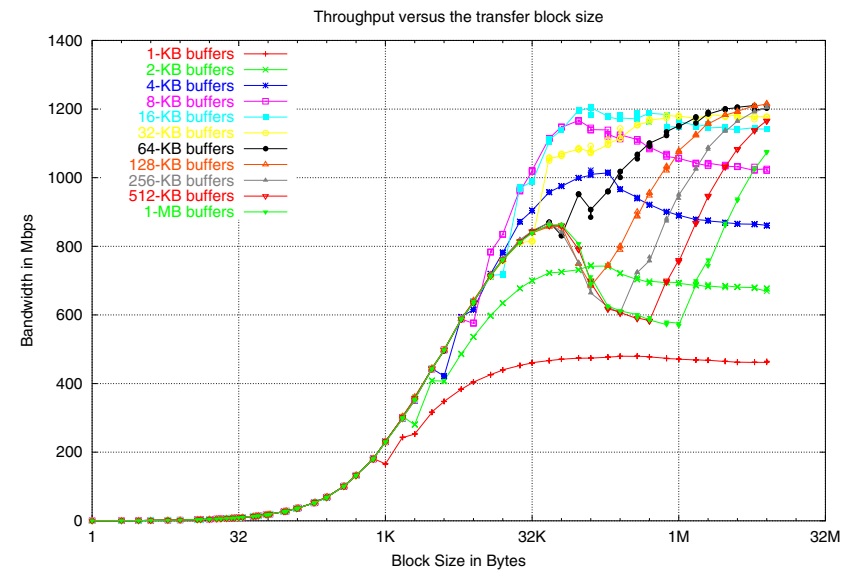

\section{Figure 5. MMP-Sockets throughput perfor- mance using 4 buffers}

even the possibility of using concurrently the four buffers is not enough for very large messages. The fragmentation, and implied saturation, creates such bottleneck to performance that even with the possibility of pipeline transmission performance does not rise to more than 700 -Mbps for the 2-KB PB size. This performance is even lower than the performance obtained with the serial transmission, by using only one large PBs. Only for PBs sizes greater than 8-KB, advantage is taken the full pipeline transmission. The lines plotted in figure 5 clearly demonstrates that the PB size influences the enabling point of pipeline transmission. From the multitude of tests performed, the sizes of $16-\mathrm{KB}$ and 32-KB show the highest average throughput. The throughput results for these two buffer sizes (16KB and 32KB) are presented in figure 6 , while minimum latency for small messages is about $25 \mu \mathrm{s}$ in both cases.

From the analysis of these results, it can be concluded that MMP-Sockets should use the quantity of fifteen buffers of 16-KBytes and, if possible, to adapt the buffer size to 32-KBytes in cases that applications request a single transmission of more than 512-KBytes. From the latency point of view, there is no significant difference between the two buffer sizes. Since using buffers with different sizes does not allow to take advantage of the strategies described in section 3.3, 16-KBytes was adopted as the optimal value for the buffer size.

\subsection{Intra-host copy operations analysis}

This experiment analyzes the cause of the drop on throughput performance around data sizes of $128-\mathrm{KB}$, when using PB larger than the transmitted data, to force serialization, as visible in figure 5 .

The transmission time or latency for a small message of size $s$ is given by the time used in the MMP transmission of the message, plus the time used in the implementation for 


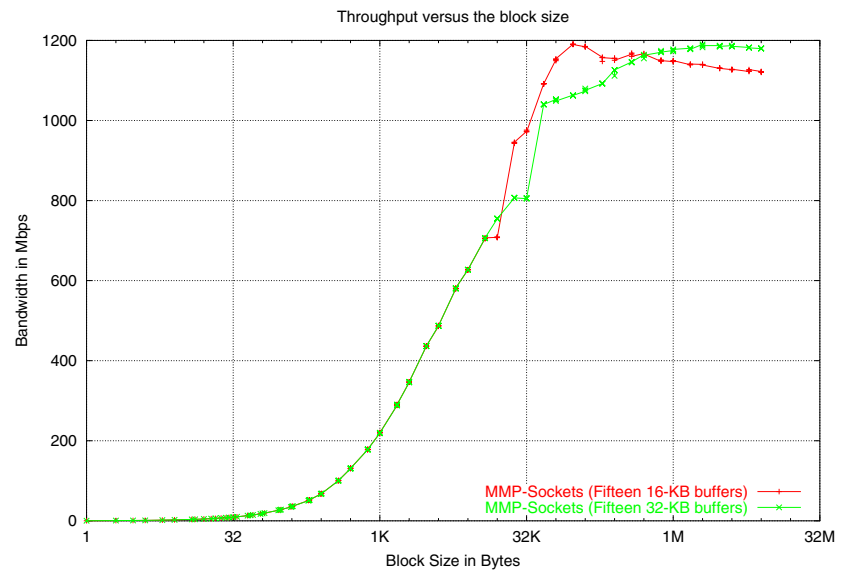

Figure 6. Throughput of MMP-Sockets for buffer sizes of 16-KB and 32-KB

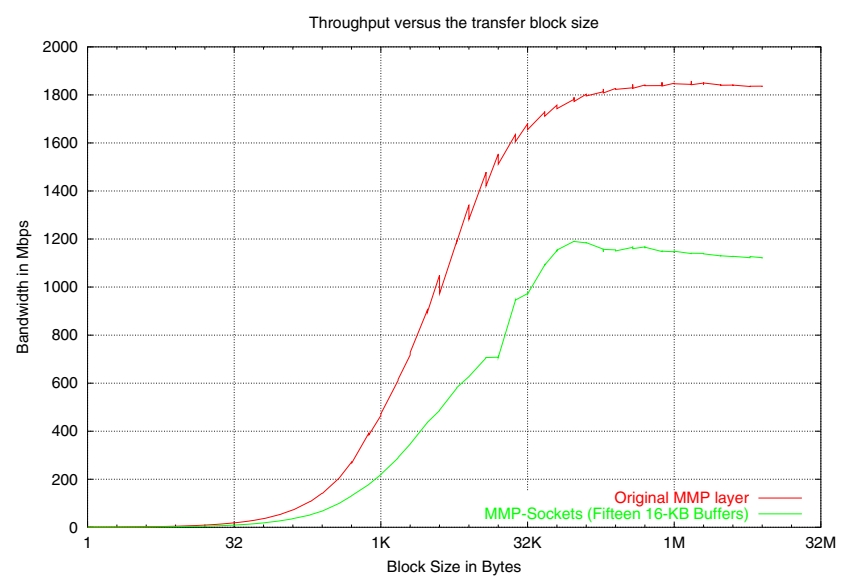

Figure 7. MMP-Sockets and original MMP performance

inherent processing by the sending and receiving nodes.

In figure 7, which presents the MMP throughput performance, the MMP transmission time grows linearly after saturation point, reverting the cause of loss of performance to the other operations in the transmission. The implementation time presents two main components: the execution of the code to process the request and the copy operation procedure between UB and the PB. Request processing operations are mainly constituted by look-up operations, so their processing overhead remains constant, whichever the transmitted size.

An analysis can be performed to compare the three time consuming terms of equation (1) below, where $T_{o}$ represents the sum of the fixed processing overhead for sender and receiver. Table 1 presents such an analysis, where $T_{c p y()}$ represents the time obtained when performing a memcpy () operation of size $s$ between PBs; $T_{\text {lat }}$ represents the time for the full transmission of the block size $s$ by the implementation, using only one PB of 1-MB to force
Table 1. Times spent in main transmissions stages with size $s$

\begin{tabular}{c|rrr|rr}
\hline $\begin{array}{c}\text { Size } s \\
\text { (Bytes) }\end{array}$ & $\begin{array}{c}T_{M M P} \\
(\mu \mathrm{s})\end{array}$ & $\begin{array}{c}T_{c p y} \\
(\mu \mathrm{s})\end{array}$ & $\begin{array}{c}T_{l a t} \\
(\mu \mathrm{s})\end{array}$ & $\frac{T_{c p y}}{T_{M M P}}$ & $\frac{2 * T_{c p y}}{T_{l a t}}$ \\
\hline 32 & 13.4 & 0.3 & 26.4 & $2 \%$ & $2 \%$ \\
$16 \mathrm{k}$ & 80.9 & 64.2 & 164.0 & $79 \%$ & $78 \%$ \\
$64 \mathrm{k}$ & 285.3 & 276.7 & 583.2 & $97 \%$ & $95 \%$ \\
$512 \mathrm{k}$ & 2188.0 & 2439.8 & 6831.4 & $112 \%$ & $71 \%$ \\
$1 \mathrm{M}$ & 4363.6 & 4901.9 & 13872.0 & $112 \%$ & $71 \%$ \\
\hline
\end{tabular}

serialization; $T_{M M P}$ in the transmission of the block size $s$ by MMP library; $\frac{T_{c p y}}{T_{M M P}}$ represents the relation between the time spent in performing the memcpy () operation and the time spent in the MMP transmission of a message with the same size, while $\frac{2 * T_{c p y}}{T_{l a t}}$ represents the ratio of the overall time spent in memcpy () operations and the transmission time using MMP-Sockets. This last column in table 1 indicates the relative importance of the memcpy () operations in the serial transmission time (1). All times were measured using NetPIPE appropriate implementation.

$T_{\text {lat }}(s)=T_{c p y}^{U B \rightarrow P B}(s)+T_{M M P}(s)+T_{c p y}^{P B \rightarrow U B}(s)+T_{o}$

The times presented in table 1 show that the time spent in the memcpy ( ) operation, performed inside the hosts involved, becomed equal or higher than the time spent in the MMP transmission for messages larger than $16-\mathrm{KB}$. This means that more than $70 \%$ of the total transmission time is spent in the copy operations inside each host.

It is from the transfer buffer size of $64-\mathrm{KB}$ that the memcpy ( ) operation starts to have an important role in the loss of performance: the time spent in the memcpy () is of the same magnitude order than the MMP transmission latency, making it approximately two thirds of the time spent in a serial transmission.

The analysis above concludes that the use of very large pindowned buffers in the interface with MMP layer should be avoided, since it leads to a set of side effects that cause a loss of performance: it serializes the main stages of the transmission, reducing the achievable performance and drop of performance caused by the memcpy ( ) operation, which has a relevant overhead for transfer sizes above 16KB.

\subsection{Performance of MMP-Sockets versus original MMP layers}

Figure 7 depicts the throughput achieved by the MMPSockets library and by the MMP communication system. For small data transfers, the transmission time doubled, making the offered performance to be reduced to about 50\% of the original one. MMP minimum latency is about $14 \mu \mathrm{s}$ and MMP-Sockets one is about $25 \mu \mathrm{s}$. When the transfer size induces the use of pipelining in the transmission, 
the performance ratio increases to $70 \%$ of the original one, and as transfer size increases even further, this ratio decays smoothly down to $60 \%$ of the original performance offered by the original MMP layer.

The observation that for small messages the performance ratio is kept at $50 \%$ is due to the fact that the time used by the MMP-Sockets has the same grow rate than the time used in the MMP transmission. Pipelining of the transmissions stages allows for a boost to $70 \%$ of the original performance, mainly due to the overlapping of the copying and transmission operations. As the transfer size increases even more, parallelization reaches its saturation point, and the performance ratio drops to $60 \%$, mostly owed to pipeline saturation. Therefore, on MMP-Sockets that is implemented over a dedicated communication library of a cluster, the software programmer should keep a message as biggest as possible for each call.

\section{Conclusion and future work}

In this paper a new communication library for connection-oriented communication over a connection-less protocol was proposed and implementation performance evaluation results are presented. The developed library, which implements the Berkeley sockets interface on top of the Maestro2 technology, increases Maestro2 cluster compatibility. Results for the new communication library evidence a significant gain in performance, compared with using the kernel system communication library (which normally uses a off-the-shelf Ethernet connection), therefore fulfilling the main goals of this research work. Experimental results show that a maximum throughput of $1250 \mathrm{Mbps}$ is achieved by applications using MMP-Sockets, with a latency of about $25 \mu \mathrm{s}$ for messages sized up to 128-Bytes. MMP-Sockets overhead over the MMP library presents a performance of about $50 \%$ to $70 \%$ of the original MMP layer. Furthermore, the compatibility with other applications that do not use the new MMP-Sockets communication library is maintained, by using the regular TCP connection. The regular connection also serves as backup connection in case the Maestro2 environment fails, making the implementation more robust.

The experience acquired from this research work enforces the following ideas: $i$ ) the strategy for fragmentation of the application data in several datagrams, it is fundamental the number and size of the datagrams depending on particular network features; $i i)$ latency is affected due to the intrinsic operations required to perform each data transmission request, e.g. look-up functions, and the number of those operations should be minimized to reduce the incurred overhead; iii) data copy operations inside the host memory should be avoided, as they are a major performance drawback, and should be addressed by using zero-copy mechanisms, such as providing the means to the network interface so that it can copy the data directly from its buffers to the application data buffers.

Towards a transparent interface to the application, it would be important also to implement the MMP-Sockets idea as a kernel module. Moreover, this would allow to overcome some limitations imposed by MMP, such as the number of applications using it being limited to the number of available MMP ports. However, it is expected that the kernel module implementation will degrade the transmission latency, due to the increase in the processing overhead, thus relegating this approach in favor of the more efficient solution of linkable library. Furthermore, the MMP-Sockets data structures and methods are prepared to support, if required, the thread programming paradigm and the datagram based connections Berkeley sockets interface functions.

\section{References}

[1] G. Brown. GM Reference Manual. Myricom, November 2002.

[2] Dave Turner. NetPIPE - Network Protocol Independent Performance Evaluator, June 2004.

[3] M. Fischer. GMSOCKS - A Direct Sockets Implementation on Myrinet. In CLUSTER '01: Proceedings of the 3rd IEEE International Conference on Cluster Computing, page 204, Washington, DC, USA, 2001. IEEE Computer Society.

[4] H. Tezuka et al. PM: a high-performance communication library for multi-user parallel environments, 1996.

[5] IEEE Computer Society. IEEE Standard for Low-Voltage Differential Signaling (LVDS) for Scalable Coherent Interface (SCI). IEEE, July 1996. IEEE Standard 1596.3-1996.

[6] Jiani Guo et al. A cluster-based active router architecture supporting video/audio stream transcoding service. In IPDPS '03: Proceedings of the 17th International Symposium on Parallel and Distributed Processing, page 44.2, Washington, DC, USA, 2003. IEEE Computer Society.

[7] Jonathan Corbet, Alessandro Rubini, Greg Kroah-Hartman. Linux Device Drivers, 3rd Edition. O'Reilly and Associates, 3 edition, February 2005.

[8] Loc Prylli et al. Modeling of a high speed network to maximize throughput performance: the experience of BIP over Myrinet. Parallel and Distributed Processing Techniques and Applications (PDPTA'98), 1998.

[9] MPI Forum. MPI: A message-passing interface standard.

[10] Myricom. Myrinet. http://www.myri.com/.

[11] Shinichi Yamagiwa et al. Design and implementation of message passing library on maestro network. In IEEE Pacific Rim Conference on Communications, Computers and Signal Processing, pages 87-90, August 2001.

[12] Shinichi Yamagiwa et al. On the performance of Maestro2 High Performance Network Equipment, Using New Improvement Techniques. In Proceedings of IEEE IPCCC2004, Phoenix, USA, April 2004.

[13] Xilinx Inc. Virtex-II Platform FPGA Data sheet, 2002. 